Original Article

\title{
Global vulnerability to near-Earth object impact
}

\author{
Nicholas J. Baileya , Graham G. Swinerd ${ }^{a}, *$, Hugh G. Lewis ${ }^{a}$ \\ and Richard Crowther ${ }^{b}$ \\ ${ }^{a}$ Astronautics Research Group, School of Engineering Sciences, University of \\ Southampton, Southampton SO17 1BJ, UK. \\ ${ }^{b}$ Rutherford Appleton Laboratory, Harwell Science and Innovation Campus, \\ Didcot, Oxon, OX11 0QX, UK.
}

*Corresponding author.

\begin{abstract}
A clear appreciation of the consequences resulting from an asteroid impact is required in order to understand the near-Earth object (NEO) hazard. Three main processes require modelling to analyse the entire impact event. These are the atmospheric entry phase, land impact events and ocean impact events. A range of impact generated effects (IGEs) are produced by different impact scenarios. It is these IGEs that present the threat to human populations worldwide, and the infrastructure they utilise. A software system for analysing the NEO threat has been developed, entitled NEOimpactor, to examine the social and economic consequences from land and ocean impacts. Existing mathematical models for the three principal impact processes have been integrated into one complete system, which has the capability to model the various effects of a terrestrial asteroid impact and, critically, predict the consequences for the global population and infrastructure. Analysis of multiple impact simulations provides a robust method for the provision of an integrated, global vulnerability assessment of the NEO hazard. The primary graphical outputs from NEOimpactor are in the form of 'relative consequence' maps, and these have been designed to be comprehensible to a non-specialist audience. By the use of a series of multiple-impact simulations, the system has identified the five countries most at risk from the impact hazard, as well as indicating the various factors influencing vulnerability.
\end{abstract}

Risk Management (2010) 12, 31-53. doi:10.1057/rm.2009.16

Keywords: near-Earth object; global hazard; global vulnerability; impact simulation 


\section{Introduction}

The primary aim of the study presented in this article is to investigate the global asteroid impact vulnerability using a new software tool. This is used to derive a global risk assessment based on the threat to human populations worldwide, and their associated infrastructure. NEOimpactor is a first step in the provision of useful tools in the planning of future impact mitigation missions, and in the decision- and policy-making activity that will be required in the event that the Earth is threatened by an impact event. It is clearly of importance that this activity be informed by reliable data regarding each country's relative vulnerability, in order to accurately understand the factors associated with a particular asteroid threat. The availability of such data, in an open international forum, will allow each nation an equal voice, and prevent the possibility that mitigation missions are planned based solely on the participants' vested interests. In the event that mitigation fails, such information would also be useful in assessing which countries require assistance.

This study focuses upon the scenario of an asteroid impacting the Earth. Such a scenario is rarely analysed as it suggests that either an undetected threat has suddenly appeared, or that the mitigation of a known threat has failed. However, there is a need to study such a scenario, as otherwise there would be a gap in our understanding of Earth's vulnerability to the asteroid hazard. Such a study also aids the planning and preparedness which governments require to improve resilience to the hazard, including coordination of both pre-evacuation and post-disaster relief efforts. It is also helpful in fully understanding the potential outcomes of a mitigation mission which might alter the predicted impact site. Furthermore, it can be used as a potential means of assessing where the responsibility for mitigation lies by identifying the nations that are most affected. At a national level, the population loss will impact on a social level. For example, this includes a reduction in the skilled workforce, so hindering the return to normality. Lost infrastructure, which will require re-building, will impact the local economy while also leading to the displacement of communities from affected homes. At an international level, it is important to identify those countries most at risk due to a random impact event, and also the vulnerability of each country.

In order to provide this risk assessment, a global study of the threat must be undertaken, as called for by Galvez et al (2003). In the case of land impacts, the impact generated effects (IGEs) of small asteroids will be relatively localised, whereas an ocean impact-generated tsunami will have the ability to propagate the impact energy across the globe. For this reason the study must be performed at a global level. Vulnerability is assessed by considering the level of destruction each country is likely to experience due to an impact and is measured in terms of the population lost (casualty estimate) and the cost of the 
infrastructure loss (damage estimate). The NEOimpactor tool allows the investigation of the following:

- Impact modelling - this includes atmospheric entry (with the associated aerodynamic effects), the land impact effects and the ocean impact effects. Each of these is implemented at a global level so the consequences from every impact on each country can be quantified.

- Population and infrastructure interactions - an assessment of the impact consequences is achieved through an examination of each IGE, and the influence it has on the local human population and infrastructure. The severity of the consequence will depend on the magnitude of the IGE. The risk assessment will be based on a summation of the casualty and damage estimates.

- Development of outputs that are readily understood - the nature of the near-Earth object (NEO) hazard, unlike other natural disasters, is that it represents a widespread threat to all people on Earth. However, the infrequency of impact events leads to general disregard of the hazard. Simulation results that are understandable by a wide section of society are vital for successful knowledge dissemination, and in terms of raising awareness of the hazard. The understanding of an individual country's vulnerability is important to help improve resilience.

- Development of a global vulnerability assessment - at an international level, each country will have a relative vulnerability associated with the NEO hazard. Assessment of the impact consequences on a global scale across multiple countries will help identify particularly vulnerable countries. Such information is required to inform the policy-making activities so that resilience can be maximised. These decisions are most powerful and influential when made through intergovernmental cooperation and organisations such as the United Nations.

\section{Methodology}

In the NEOimpactor simulation, the asteroid is modelled from atmospheric entry to the ground. Both land and ocean impact scenarios are included, using the results from current literature. The various IGEs are modelled, and their consequences upon the human population and associated infrastructure are assessed. Atmospheric passage is modelled by a propagator that takes account of the Earth's gravitational acceleration and atmospheric drag. Given the short duration of atmospheric passage, a fourth-order Runge-Kutta integrator is adequate to predict the object's path. Frictional heating leads to out-gassing, combustion and vaporisation of the surface material of the impactor over time, so that mass is lost by the process of ablation. NEOimpactor implements the two-stage regime outlined in Chyba (1993). In general, the lack of 
experimental data related to the asteroid's atmospheric passage inhibits understanding of the processes involved. However, the software architecture allows each model to be enhanced easily, when experimental data become available.

In this process, we adopt object radius as a convenient proxy for impact energy, which is a common practice. However, it is important to note that it actually the impact energy that is the principal driver in determining the impact consequences. The impact energy associated with a local scale event $(25 \mathrm{~m}$ radius sphere with a speed of $12000 \mathrm{~m} / \mathrm{s}$ and density of $3500 \mathrm{~kg} / \mathrm{m}^{3}$ ) is of the order of $1.65 \times 10^{16} \mathrm{~J}$. It is easily demonstrated that this energy can be achieved by objects of various sizes and speeds. Results are also presented for the regional $(125 \mathrm{~m}$ radius object) and global (225 $\mathrm{m}$ radius object) scale events. For ease of understanding, we continue to use the commonly used practice of describing the outcome of the simulations in terms of object size, despite its shortcomings.

The range of asteroid radii studied by NEOimpactor is limited to between 25 and $250 \mathrm{~m}$. Note that this is the size of the object after atmospheric passage. This range accounts only for a subset of the orbital asteroid population. It falls below the Spaceguard Survey study range of $>500 \mathrm{~m}$ radius (Stokes and Yeomans, 2003) - a size at which the impact consequences become catastrophic. Objects in NEOimpactor's study range remain both largely undetected and frequently unconsidered in the literature. In all the cases investigated in this study, the asteroid's other parameters, such as velocity, density and shape, have been fixed allowing radius to be used as a proxy for kinetic energy. The velocity chosen, $12000 \mathrm{~m} / \mathrm{s}$, is at the lower end of the impact speed range and the object's density, $3500 \mathrm{~kg} / \mathrm{m}^{3}$, is at the upper end of the density range. As such, the results should provide a reasonable representation of the impact consequences. The asteroid composition assumed throughout is a stony iron. Asteroids comprised of this material are more likely to reach the Earth's surface intact.

Three event scale classifications are used to aid in the interpretation of the results. Each of these is identified with a particular size of object - 25, 125 and $225 \mathrm{~m}$ radius - as mentioned above. The classification is summarised as:

- Local: Events that affect an area typically smaller than a country.

- Regional: Events that affect one country severely and possibly its neighbours.

- Global: Events that have a significant effect on many countries.

\section{Land impacts}

If the impact site is located on land, NEOimpactor initially models the generation of the impact crater and subsequently the IGEs - ejecta material, thermal flux from the fireball, the atmospheric blast wave and the seismic shock. The models for these IGEs are largely based on the comprehensive work of Collins et al (2005), 
which predict the magnitude of each effect at a specified distance from the impact site:

- Crater - the impact crater is generated in two stages. The impact energy initially excavates a 'transient' crater. This is a simple parabolic-shaped depression, the material from which is thrown into the atmosphere. As this excavated material lands, and the seismic energy dissipates, the final crater shape emerges.

- Ejecta - this material is initially excavated by the impact energy, and as such the volume of ejecta is equal to the transient crater volume. Much of the material falls to Earth within the boundary of the transient crater. However, the remainder is thrown radially from the impact site onto the surrounding terrain.

- Fireball - the impact explosion generates a fireball that expands and rises above the impact site. Initially the temperature is such that the fireball is opaque, and the thermal radiation is confined. However, as it expands and cools, the fireball drops below the opaque transition temperature, at which point the peak level of radiation is released.

- Blastwave - the impact explosion generates a sudden expansion and compression of the local atmosphere. This results in a pressure blast wave which propagates out from the impact site. The maximum pressure difference across the blast wave, called the peak over-pressure, is the critical parameter defining the wave's destructive potential. As the energy of an impact explosion is many orders of magnitude larger than those generated by explosives, Collins et al (2005) use a scaling rule referenced to a 1 kiloton explosion.

- Shockwave - the impact event releases a significant amount of energy into the ground as seismic shock waves. These can be equated to typical geological earthquake events measured using the Richter scale. This magnitude is used to assess the structural damage sustained at each point on the Earth's surface, referenced to the equivalent damage sustained by geological earthquakes.

\section{Ocean impacts}

Work by Ward (2000) is used as the basis for the tsunami model. The tsunami wave that results is dependent upon the characteristics of the initial ocean cavity excavated by the impact. Following the initial definition of the cavity, the wave is propagated across the ocean. The ocean is modelled as a cellular grid with equal latitude and longitude bins. The model algorithm propagates the wave across the cell boundaries and, in an iterative manner, radiates the wave out in one cell steps. During deep water propagation the algorithm uses linear theory to estimate the current wave speed, amplitude and critical depth. The wave amplitude in deep water is based upon the dimensions of the initial cavity created by the impact energy (Ward and Asphaug, 2002). It is the in-filling of this cavity which sets up the ocean oscillation which generates the tsunami wave. 
Ocean depth information determines whether the wave has a deep water wave characteristic, or if it begins to shoal. These depth data are extracted from a global bathymetric data set. The limitation of this algorithm is the inability of the wave to interact with itself to exhibit interference, as only the single peak wave amplitude is modelled. However, unlike ray-tracing methods, this algorithm successfully deflects the wave around complex coastlines to eventually shoal on every coastal shoreline, and so, to some extent, simulates diffraction effects. Eventually the wave reaches shallow water and begins to build in amplitude and break. The distance inland that the wave reaches is called the run-in distance. NEOimpactor determines how much of the coastal cell is inundated. The associated term run-up defines how far vertically the wave will reach. If the coastline is raised above this level then the location will be safe from inundation. Run-up is calculated directly from work by Ward and Asphaug (2003).

\section{System overview}

NEOimpactor adopts a modular system architecture with three primary components:

- the User Interface (UI),

- Simulation Applications (SAs),

- Operational Modules (OMs).

These are illustrated as a combined Venn diagram in Figure 1. The entire $\mathrm{C}++$ application was developed using the Microsoft Visual Studio software package, as this gives access to the Microsoft Foundation Class (MFC) environment. This MFC environment handles the creation of the familiar windows user interface systems, and this is how the NEOimpactor UI is organised. Via the UI, the

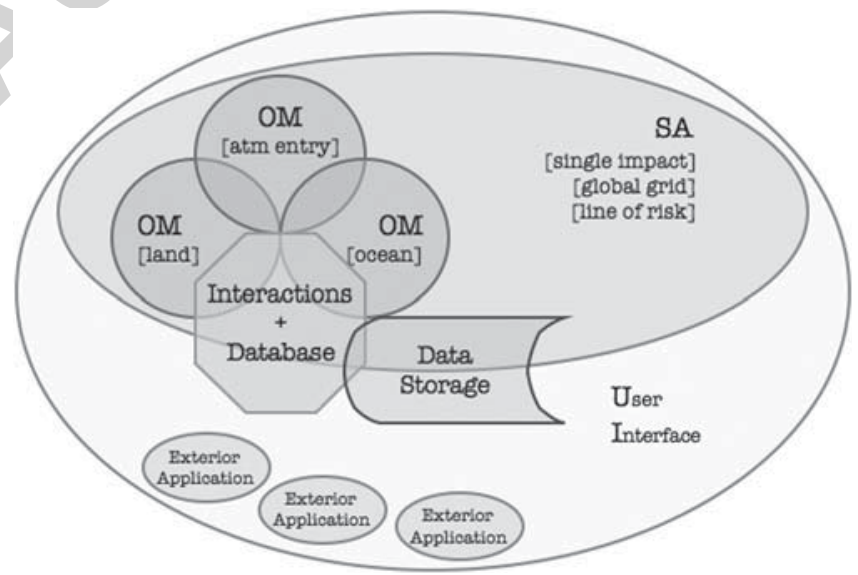

Figure 1: Venn diagram of the NEOimpactor system overview. 
user is able to select and run one of the main SAs, or to choose from a set of independent 'applets' (small exterior applications). It is the SAs that handle the OMs, which contain the mathematical models. The large amount of terrestrial data required to manage the process is held by a database model of the Earth, from which output data can be extracted and saved to the user's hard disk.

From the UI menu options, the user selects one of three SAs: 'Single Impact', 'Global Grid' or 'Line of Risk'. Once launched, the SA runs independently of the user, handling the subsequent processes autonomously. Initially, the applications require starting conditions and simulation limits, which are input by the user utilising dialogue boxes. These data are passed to each OM in turn, as well as being stored as text files on the hard disk. Manipulation of the data defining the asteroid is performed by the OM. Data are passed from the atmospheric flight analysis to the relevant impact OM which models the IGEs. Interaction of the IGEs with the Earth is then handled by the database module.

At the heart of the software is the system database, which offers rapid access to layered data and enables cross referencing of layers to generate the results. Unlike the OMs, which work on a single flow of data (such as, for example, modelling the flow of kinetic energy through the impact event), the database $\mathrm{OM}$ stores and retrieves data and handles data queries. The database structure resembles a rectangular map of the Earth, with each cell having a specific dimension in latitude and longitude. Each layer of the database records a single characteristic for that cell, such as ocean depth or population count. Queries access different layers to extract particular data sets, and entire layers can be extracted in raster form (a pixel image) as a global map.

The final stage of operation is data storage. NEOimpactor constructs a directory tree for the data output, storing the results from each SA together in one directory. The results from each run of the SA are stored in sub-folders labelled by simulation name and date to distinguish them, and to facilitate ease of data retrieval. The output data are generated in three forms: as text files (for example, the characteristics of the chosen asteroid), as raw data files (in '.csv' format, listing the consequences for each cell of the database) and as impact maps (using the 'parallel projection' cartographic projection). The digital originals can also be imported into other software packages such as Google Earth.

\section{The global grid simulation application}

The Global Grid SA is the most complex simulation. The aim of the Global Grid is to investigate the relationship between the impact consequences and impact location. This is achieved by simulating an asteroid impact at multiple, uniformly spaced locations to identify geographical regions where the consequences are most severe. This is done by dividing the globe into a series of 'grid cells' (the 'Global Grid' is of a lower resolution grid than the database grid), into which the same impact event is simulated. The consequences are then recorded for each grid cell impact. The choice of grid resolution is a trade-off 
between runtime and result data quality. Using a dual core $2.2 \mathrm{GHz}$ AMD processor based system, the maximum Global Grid resolution simulation achievable, corresponding to the resolution of the database, would take approximately four years of processing time.

The impact co-ordinates are taken as the central co-ordinates of each grid cell. The same impact event is simulated in each grid cell by the relevant OM and the casualty and damage consequences are recorded. A global map is constructed by the Global Grid SA by shading each grid cell according to the severity of the impact consequences. Examples of the output of this SA are shown in Figure 2.

\section{The 'line of risk' simulation application}

This application operates as a one-dimensional version of the Global Grid model. While the Global Grid generates a two-dimensional array of identical impacts, this application places impacts along a one-dimensional line. The user chooses either a path along a particular line of latitude or longitude, or a path between two locations on the Earth. The resolution of the line governs how many impacts will be simulated, with the same trade-off between runtime and accuracy. The advantage of running a line impact simulation is that higher resolution paths are achievable, as runtime scales linearly with the resolution in this case. The output of the SA is a list of casualty and damage estimates for impacts simulated along the line. These data can then be plotted to highlight the sections of the path which generate the most significant consequences. In the event of a real threat, the measurement uncertainties in an asteroid's orbit will produce a line of risk on the Earth, such as that predicted for the asteroid 99942 Apophis' 2036 transit. This path can be imported into NEOimpactor to find the associated risks along the path.

\section{Sources of error in the NEOimpactor system}

Within the model itself a number of sources of error can be identified. The greatest of these is the simplification in the overall modelling of such a complex event, combined with the global nature of the investigation. At each stage of the process, the models used are approximations. While hydrocode simulations can model the complex behaviour associated with impact events with a greater degree of accuracy, their long processing times and high computational demands make them unfeasible for application to the global problem. Furthermore, investigating the threat to the Earth as a whole prevents any detailed study of the consequences for specific local regions. In addition, the simplification of the data model representing the characteristics of the Earth will naturally be another source of error. So while the absolute estimates of specific outcomes cannot be taken as accurate predictions of the real consequences, it is important to emphasise that the overall global vulnerability can only be studied in relative terms. 

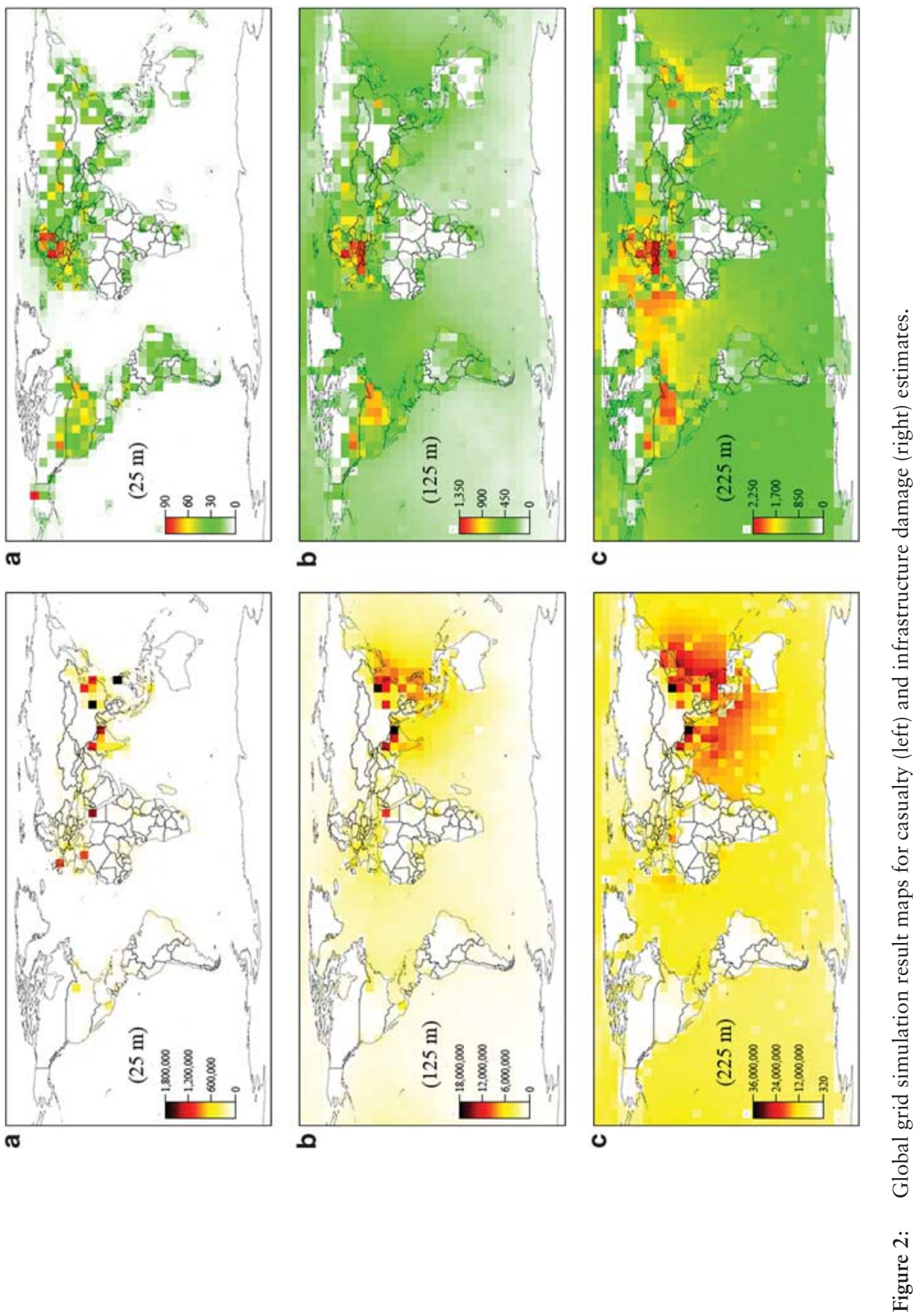


\section{Results}

\section{Global grid casualty results}

Figure 2 (left) displays the casualty result maps for the three object sizes -25 , 125 and $225 \mathrm{~m}$. For the local event ( $25 \mathrm{~m}$ radius), two peak cells are identified, one in central China and the other in the Philippines. A few other cells are highlighted across India, North Africa and Europe. Most of the ocean cells appear white, indicating the low potential these impacts have for generating casualties. Comparatively the regional event $(125 \mathrm{~m}$ radius) chart presents a concentration of highlighted land cells in India and Southeast Asia, as well as a significant increase in the danger from ocean impacts. The global event ( $225 \mathrm{~m}$ radius) map clearly demonstrates the significance of ocean impacts to the generation of casualties, with all ocean impacts producing significant casualty estimates.

Data extracted from the simulation are summarised in Table 1 . This gives the impact consequences for the 250 countries in the NEOimpactor database.

Table 1: Analysis of the casualty data drawn from the three Global Grid simulations depicted in Figure 2

\begin{tabular}{|c|c|c|c|}
\hline & Local & Regional & Global \\
\hline \multicolumn{4}{|l|}{ Peak impact data } \\
\hline Type & Land & Land & Land \\
\hline Country & China & $\begin{array}{c}\text { India, } \\
\text { Bangladesh } \\
\text { and Nepal }\end{array}$ & China \\
\hline Latitude & $30.9^{\circ} \mathrm{N}$ & $25.3^{\circ} \mathrm{N}$ & $36.6^{\circ} \mathrm{N}$ \\
\hline Longitude & $104.1^{\circ} \mathrm{E}$ & $87.2^{\circ} \mathrm{E}$ & $115.3^{\circ} \mathrm{E}$ \\
\hline Casualty estimate & 1688450 & 16981700 & 34741700 \\
\hline \multicolumn{4}{|l|}{ Most affected country } \\
\hline Averaged over grid & China & China & China \\
\hline Casualties per impact & 4042 & 139567 & 814085 \\
\hline \multicolumn{4}{|c|}{ No. of countries suffering casualties (\%) } \\
\hline$>1000$ & 0.8 & 32.8 & 56.8 \\
\hline$>10000$ & 0.0 & 7.6 & 28.4 \\
\hline$>100000$ & 0.0 & 0.8 & 5.6 \\
\hline \multicolumn{4}{|c|}{ No. of impacts generating casualties (\%) } \\
\hline$>100000$ & 3.9 & 77.0 & 81.3 \\
\hline$>500000$ & 0.5 & 50.5 & 76.7 \\
\hline$>1000000$ & 0.3 & 31.6 & 74.3 \\
\hline \multicolumn{4}{|l|}{ Average no. of casualties } \\
\hline Ocean impacts & 3518 & 849732 & 6167927 \\
\hline Land impacts & 13725 & 207760 & 438703 \\
\hline All impacts & 17243 & 1057492 & 6606630 \\
\hline
\end{tabular}


The peak value for each simulation is either in China or India. However, in all three cases (local, regional and global), summing the casualty figures over the entire grid reveals that the most affected country per impact is China. Finally the table provides some grid-average values to compare results for each object size. The ocean and land impacts show a transition from land-dominance to ocean-dominance as the object size increases.

\section{Global grid infrastructure damage results}

Figure 2 (right) shows the equivalent infrastructure damage distribution maps generated during the simulation. In the model, night-time light pollution is used as a proxy measurement for infrastructure density (NASA, 2008). The monetary cost of the damage is expressed in 'NEOimpactor Infrastructure Units' (NIU), where $1 \mathrm{NIU}$ is equivalent to approximately US\$0.2 billion. In comparison to the casualty data maps, these results predominantly highlight regions in Europe and North America as being most at risk. This is to be expected, due to the large areas of high infrastructure density in these regions. The local scale event ( $25 \mathrm{~m}$ radius object) map indicates that land impacts are most significant, with all impacts likely to generate infrastructure damage except in regions of Canada, Africa, Russia and Australia. However, as the asteroid size increases, ocean impacts become increasingly dominant.

Table 2 summarises the infrastructure damage simulation data. Overall, the United States suffers the greatest infrastructure loss, due to the high density of infrastructure along the US Eastern seaboard. The comparison of all averaged ocean and land impacts shows the same transition from land to oceandominance as the object size increases.

\section{Presentation of country-by-country summed data}

NEOimpactor records the impact consequences attributed to each country in the database. Thus for each simulation, the severity of the consequences for every country can be determined. NEOimpactor generates an output map using this data, shading each country according to its cumulative consequence estimate. While each of the 250 countries modelled could be shaded separately, a five-colour choropleth map format is adopted. This groups countries based on the severity of the impact consequences, allowing an alternative representation of the results. An example of this output map format is given in Figure 3, using data from the $125 \mathrm{~m}$ radius object simulation. The advantage of this format is that, in one single image, the data for every country are presented.

\section{Global ranking based on overall casualty and damage sustained}

In Table 3 the cumulative casualty and damage data are presented for the three event scale simulations. These results show those countries that face the 
Table 2: Analysis of the damage data drawn from the three Global Grid simulations depicted in Figure 2

\begin{tabular}{|c|c|c|c|}
\hline & Local & Regional & Global \\
\hline \multicolumn{4}{|l|}{ Peak impact data } \\
\hline Value (NIU) & 92.5 & 1325 & 2556 \\
\hline Type & Land & Land & Land \\
\hline Country & Sweden & Sweden & $\begin{array}{c}\text { Germany, } \\
\text { Austria, Czech Rep }\end{array}$ \\
\hline Latitude & $59.1^{\circ} \mathrm{N}$ & $59.1^{\circ} \mathrm{N}$ & $47.8^{\circ} \mathrm{N}$ \\
\hline Longitude & $14.1^{\circ} \mathrm{E}$ & $14.1^{\circ} \mathrm{E}$ & $14.1^{\circ} \mathrm{E}$ \\
\hline \multicolumn{4}{|l|}{ Most affected country } \\
\hline Averaged over grid & US & US & US \\
\hline Average damage (NIU) & 4 & 22 & 79.5 \\
\hline \multicolumn{4}{|c|}{ No. of countries suffering damage (\%) } \\
\hline$>0.5 \mathrm{NIU}$ & 0.8 & 19.6 & 46.4 \\
\hline$>5 \mathrm{NIU}$ & 0.0 & 4.0 & 14.4 \\
\hline$>50 \mathrm{NIU}$ & 0.0 & 0.0 & 0.4 \\
\hline \multicolumn{4}{|c|}{ No. of impacts generating damage (\%) } \\
\hline$>5 \mathrm{NIU}$ & 14.7 & 83.5 & 85.6 \\
\hline$>50 \mathrm{NIU}$ & 2.4 & 77.3 & 82.7 \\
\hline$>500 \mathrm{NIU}$ & 0.0 & 7.8 & 74.0 \\
\hline \multicolumn{4}{|l|}{ Average damage } \\
\hline Ocean impacts (NIU) & 0.5 & 125 & 692 \\
\hline Land impacts (NIU) & 4 & 65.5 & 138 \\
\hline All impacts (NIU) & 4.5 & 190 & 830 \\
\hline
\end{tabular}

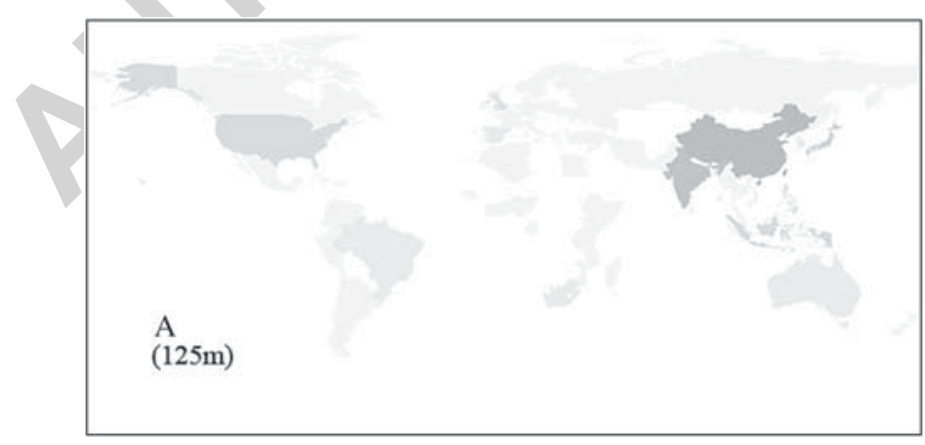

Figure 3: The cumulative consequence data, country-by-country, for the regional scale event (125 $\mathrm{m}$ radius object) simulation.

greatest overall global risk. The high correlation across the rankings is an indication of the stability of this ranking system. China faces the greatest risk to its population, while the US infrastructure is most at risk. 


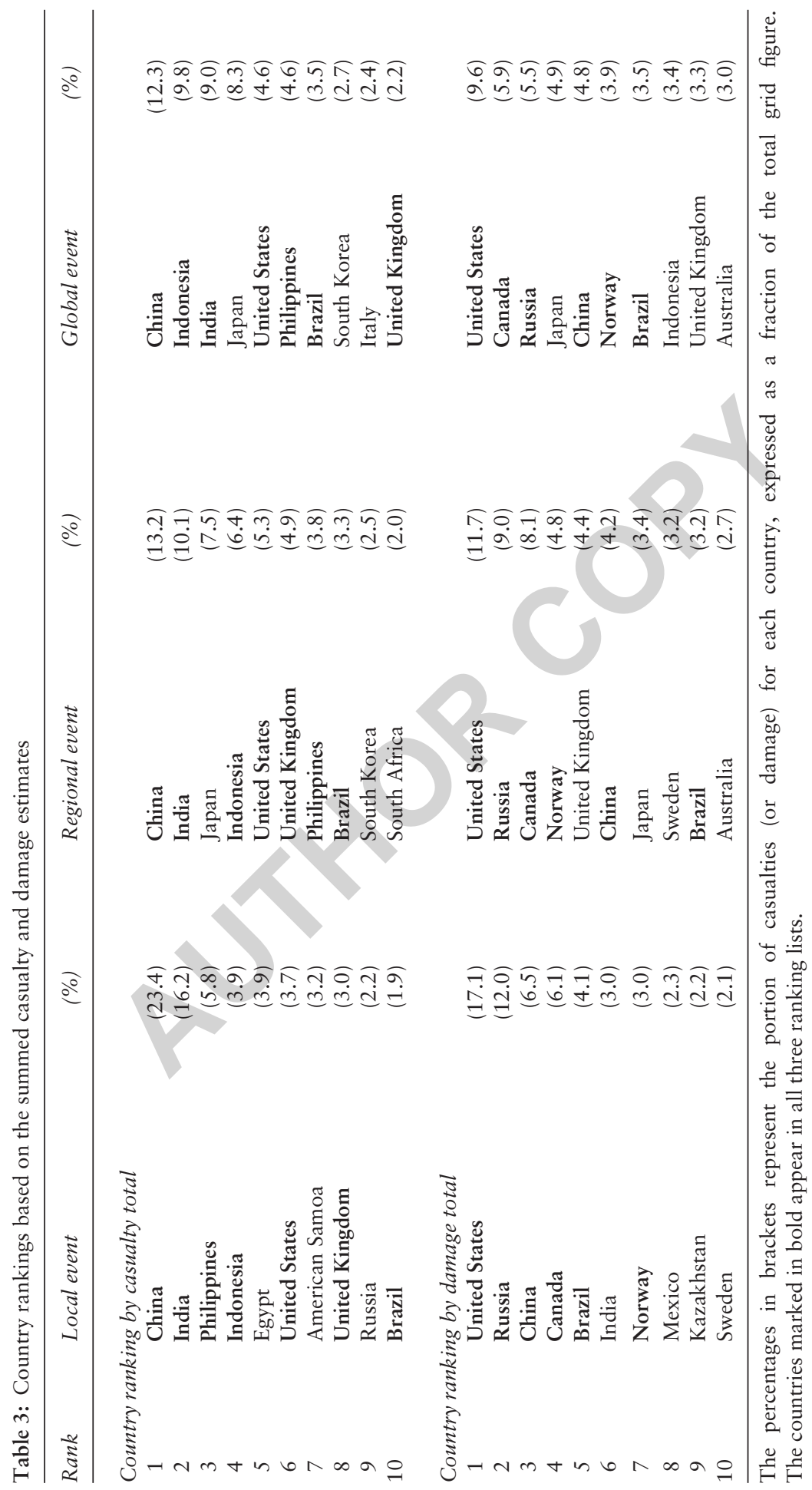




\section{Land-ocean impact severity transition radius}

The shift in the magnitude of the consequence from land impacts to ocean impacts with increasing object size is an important factor in understanding the NEO hazard. The size of object at which this switch in the severity occurs is termed the transition radius. The investigation of this characteristic must be assessed on a global basis, so data from the Global Grid simulations are used. However, it should be emphasised, once again, that we have used object size as a substitute for impact energy, and it is the energy that is the true driver in terms of the impact consequences. Hence, the simulations performed in this study are not adequate to definitively quantify the transition radius. However, as has been said above, the results of the simulations presented here are based upon spherical objects, with a larger than the average density of $3500 \mathrm{~kg} / \mathrm{m}^{3}$ and a lower than average impact speed of $12000 \mathrm{~m} / \mathrm{s}$. In light of this, it is perhaps worth analysing the results to give a rough indication of the value of the transition radius. Figure 4 displays the land and ocean data plotted against
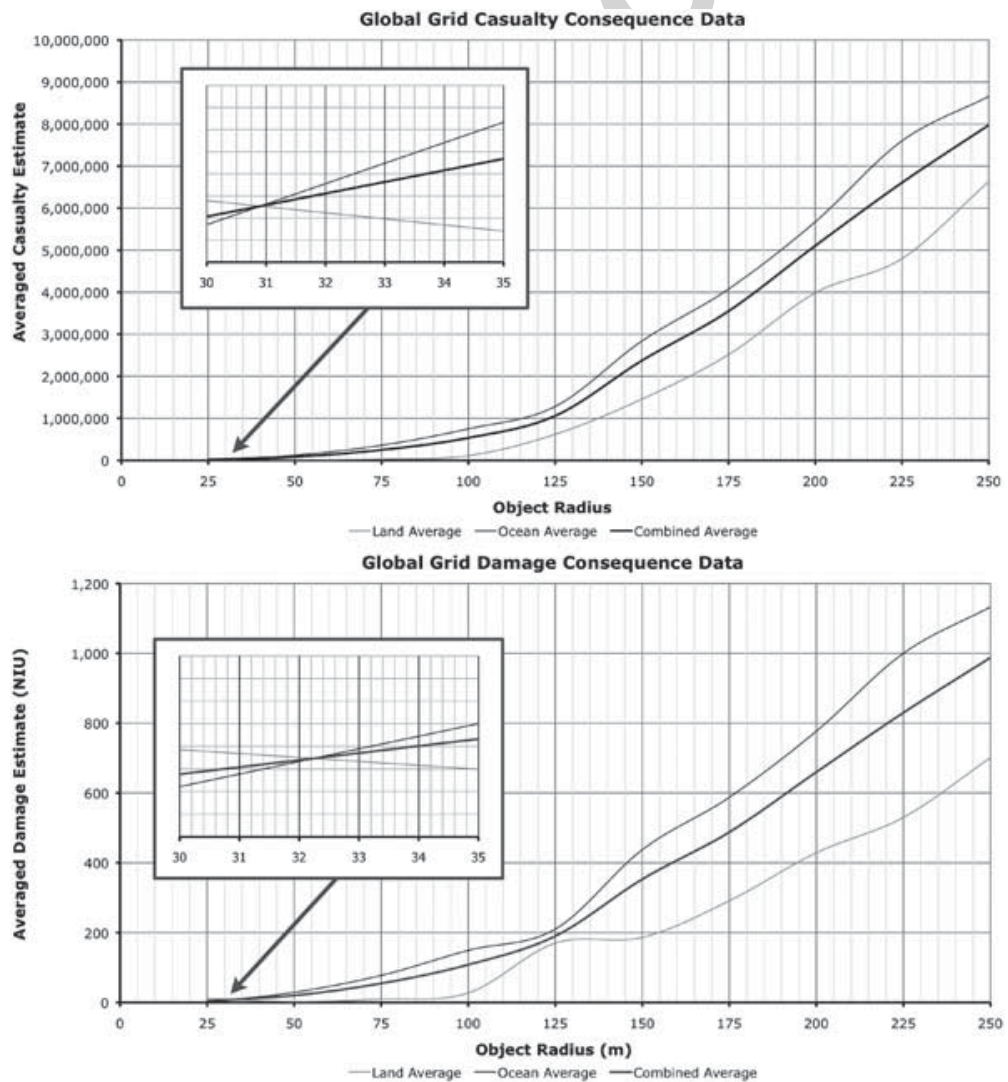

Figure 4: Land and ocean casualty (top) and damage (bottom) data plotted against object radius, showing the Global Grid transition radius as inset figures. 
object radius, with inset figures locating the transition point. By averaging the data over the entire grid, the skewing affect of particular peak impact values is reduced. These plots reveal similar values of the transition radius based on casualty and damage data -31 and $32 \mathrm{~m}$, respectively. The plots in Figure 4 also reveal that the $125 \mathrm{~m}$ radius on both the casualty and damage plots is a point where the gradient of the curves become steeper. This allows the casualty estimate and the damage estimate (in NIU) to be approximated by two line segments represented by the following equations, where $R_{N E O}$ is the asteroid radius in metres,

$$
\text { Casualty estimate }=\left\{\begin{array}{c}
10000 R_{N E O}-250000 \text { for } 25<R_{N E O}<125 \\
56000 R_{N E O}-6000000 \text { for } 125<R_{N E O}<225
\end{array}\right.
$$

and

$$
\text { Damage estimate }=\left\{\begin{array}{c}
2.0 R_{N E O}-50 \text { for } 25<R_{N E O}<125 \\
6.4 R_{N E O}-600 \text { for } 125<R_{N E O}<225
\end{array}\right.
$$

\section{The influence of fragmentation on the NEO threat to Earth}

The NEOimpactor results presented so far concern the impact of monolithic asteroids. In other words, we are adopting the assumption that the body reaches the ground intact. Pravec and Harris (2000) suggest that this assumption is valid for small bodies, whereas larger bodies (of the order of $500 \mathrm{~m}$ radius) are more likely to be loosely cohered rubble piles. However, Boslough and Crawford (2008) suggest that it is the smaller objects which fragment most easily.

Fragmentation is an important issue because of the likelihood that real bodies will fragment before impact. An understanding of the consequences of the impact of a fragmented body is also important in consideration of mitigation proposals, often involving the use of nuclear weapons, which rely on prefragmentation of the asteroid while it is still in solar orbit. The NEOimpactor fragmentation simulation has not yet been fully implemented due to difficulties in reliably modelling the processes involved. However, a simplified study is possible using the existing software by analysing the potential consequences from multiple single impacts. In this simulation, the consequences of a $250 \mathrm{~m}$ radius asteroid impact are contrasted with the impact of a number of small bodies of equivalent total mass. One thousand $25 \mathrm{~m}$ radius bodies represent an equivalent impacting mass and, with the same impact speed, an equivalent magnitude of impact energy. Table 4 presents this comparison based on Global Grid averages. This example gives a doubling of the average casualty 
Table 4: Analysis of the average impact consequences of a single impact event compared to multiple smaller impacts with equivalent impact energy

\begin{tabular}{lccccc}
\hline Impact type & \multicolumn{2}{c}{ Single 250 m radius body } & & \multicolumn{2}{c}{1000 fragments of 25 m radius } \\
\cline { 2 - 3 } \cline { 5 - 6 } & Casualties & Damage & & Casualties & Damage \\
\hline Global average & 7976483 & 987.5 & & 17243000 & 4425 \\
Land impacts & 6632036 & 701.0 & & 40796000 & 11451 \\
Ocean impacts & 8658105 & 1133 & & 5302000 & 862.5 \\
\hline
\end{tabular}

estimate and a five-fold increase in damage potential resulting from the multiple fragment impacts.

This conclusion is particularly relevant when considering mitigation mission proposals which aim to remove the threat of an approaching asteroid by disrupting the asteroid catastrophically, usually by means of nuclear detonation. The aim of such missions would be to reduce the asteroid to fragments small enough to ensure complete attenuation in the atmosphere. However, if the mission fails to achieve this and instead generates a number of bodies just large enough to reach the ground, this study suggests that the impact threat may be dramatically increased. This example therefore provides a clear argument against mitigation missions using nuclear detonation techniques.

Furthermore by taking the fragmentation results and considering land and ocean impact events separately, a second insight is revealed in Table 4. This exhibits significant increases in casualty and damage potential when an object fragments over land. On the other hand, if the fragments fall into the ocean, the consequences are reduced. This conclusion has particular relevance in understanding the threat posed by a specific asteroid, if it is expected to fragment.

\section{Discussion}

\section{Country-by-country vulnerabilty}

Seven countries appear consistently in the casualty rankings (Table 3), in spite of the correspondence between the event magnitude and the estimated losses, suggesting that particular geographic factors influence the vulnerability of a country's population. If the rankings are aggregated across all event magnitudes (as detailed in Table 5) it becomes apparent that the three top-ranked countries for casualties, China, India and Indonesia, are all situated in South and South East Asia, a region previously indicated as at risk in Figure 2. Furthermore, they have the first, second and fourth largest human populations of all countries in the database. The United States is ranked as the fourth country most at risk. Its high risk is a result of three key factors. The US population is the third highest in the world. Secondly, it has a large land area (6.4 per cent 
Table 5: The top-ten ranking by casualty and damage based on an aggregate of the three rankings given in Table 3, and the top five countries facing the greatest overall risk (right-hand column)

\begin{tabular}{lll}
\hline Casualty rank & Damage rank & Combined \\
\hline China $^{+5}$ & United States $^{\oplus, G 8}$ & China $^{+5}$ \\
India $^{+5}$ & Russia $^{G 8}$ & United States $^{\oplus, G 8}$ \\
Indonesia $^{\oplus}$ Canada $^{\oplus, G 8}$ & India $^{+5}$ \\
United States $^{\oplus, G 8}$ & China $^{+5}$ & Japan $^{\oplus, G 8}$ \\
Philippines & Norway $^{\oplus}$ & Brazil $^{+5}$ \\
Japan $^{\oplus, G 8}$ & Brazil $^{+5}$ & - \\
United Kingdom $^{\oplus, G 8}$ & Japan $^{\oplus, G 8}$ & - \\
Brazil $^{+5}$ & Sweden $^{\oplus}$ & - \\
South Korea $^{\oplus}$ & Mexico $^{+5}$ & - \\
Spain $^{\oplus}$ & India $^{+5}$ & - \\
\hline
\end{tabular}

Member countries of the Organisation for Economic Co-Operation and Development (OECD) are indicated by ${ }^{\oplus}$. Those that are part of the Group of Eight (G8) countries are indicated by ${ }^{G 8}$, while those denoted by ${ }^{+5}$ are members of the additional five countries added to the G8.

of the world's land area), and thirdly, and most significantly, the United States is exposed on both its East and West coasts to expanses of ocean. Mexico, which shares a similar geographical situation, is highlighted in the infrastructure damage ranking due to its exposure to the Pacific Ocean and the Gulf of Mexico. The Gulf itself is shallow, which reduces the peak wave height of any impact-induced tsunami, thereby mitigating some of the risk. However, evidence presented by Maurrasse and Sen (1991) and Smit (1999) reveals that the Yucatan impact, 65 million years ago, generated a significant tsunami in the Gulf which inundated the surrounding coastlines and physically altered the coastline. Furthermore, as indicated by the Mexico earthquake of 1985 in which 10000 people died, Mexico City is particularly vulnerable to earthquake destruction due to its construction on a dry lake-bed (Seed et al, 1988).

Two main factors are therefore at work in determining the vulnerability of a country's population - the size of the population at risk, and the exposure of the country to tsunami inundation. Both China and India have long coastal regions and a high proportion of coastal dwellers. China also has many highly populated port conurbations, most notably Shanghai, while India has a huge dependence on many small coastal fishing communities. The island nations of Indonesia and the Philippines suffer from the 'archipelago effect', where all populations are located close to the coast, and thus are at high risk from tsunami inundation by impacts in the surrounding ocean. This risk to Indonesia was demonstrated disastrously during the 2004 Boxing Day quake-induced tsunami, for which the greatest loss of life occurred in Sumatra (Titov et al, 2005). The Philippines have also witnessed a devastating tsunami when the volcano Pinatubo erupted and partially collapsed into the sea in 1991 (Rantucci, 1995).

The remainder of the countries listed display a varied range of vulnerability factors. The dominating factor is their coastal properties. Japan and the United 
Kingdom appear highly ranked due to the 'island effect', where many people are located in coastal communities. Being islands, they are exposed to tsunamis from every direction, although Japan's proximity to the Eurasia land mass results in the primary threat coming from the Pacific Ocean to the Southeast. The principal tsunami threat to the United Kingdom arises from impacts into the North Atlantic Ocean. South Korea, while not an island, is surrounded by ocean on three sides and faces a similar risk to that of Japan. Its geography is also similar to Japan, and has the two major ports of Busan and Pohang on its Southeast coastline, exposed to the Pacific Ocean. Coastal properties are also important factors for the remaining countries listed in Table 3. The United States, Brazil, South Korea, China and India have long coastal regions facing large oceans, and a high proportion of coastal dwellers. An understanding of the infrastructure damage rankings (Table 3 ) is aided by the same underlying rules, with the United States, presently the leading industrialised nation of the world, ranked number one.

In Table 5, the data for all scales of events are aggregated to produce a 'topten' ranking for casualties (first column) and infrastructure damage (second column). The third column in the table identifies a 'top five' nations, combining the data for both casualties and damage. In terms of the infrastructure damage ranking in Table 5, key factors that influence vulnerability to NEO impacts are the state of industrialisation of a country and the land area that is exposed to an impact. The United States, which is the leading industrialised nation in the world and occupies 6.4 per cent of the world's land area, is ranked number one based on losses to infrastructure. In addition, Russia and Canada, both developed and large nations, appear second and third. While China is demoted to fourth place, two new entries in the damage ranking are Norway and Sweden. Though not leading superpowers, these are two highly developed countries with strong infrastructure networks. One indication of this is the percentage of the population with access to broadband internet, 85 per cent compared with only 19 per cent in the United States (Savage and Waldman, 2005). Asian countries do not appear high in the infrastructure damage rankings. This is primarily due to the lower state of industrialisation in these countries. However, the lower infrastructure loss here does not equate to a lack of suffering, as the infrastructure lost represents vital services such as shelter and fresh water supplies. This is a limitation of the NEOimpactor system, as it values industrialised infrastructure above basic housing due to the way it is defined by an increased light pollution signature. Three years after the Boxing Day 2004 tsunami, Robinson and Jarvie (2008) report that coastal populations along Sri Lanka's Eastern seaboard had still to return to full function. Likewise, three years on from hurricane Katrina, large sections of New Orleans remain uninhabitable (Paxson and Rouse, 2008).

Table 5 also highlights countries that are members of the Organisation for Economic Co-operation and Development (OECD, 2009), the G8 (Group of 
Eight) and the extended G8+5. Half of the G8 countries and four of the five G8 +5 countries appear, while nearly a third of the OECD countries are listed. Crucially, only two countries listed are not members of these bodies: Indonesia and the Philippines. The OECD, G8 and G8 + 5 organisations have been established to improve communication between the most developed countries of the world. The fact that many of their members are shown to be most vulnerable to asteroid impacts is important because these countries collectively account for the majority of scientific and economic power in the world and are therefore best placed to tackle the NEO hazard. However, countries that do not possess adequate resources to rebuild following an impact need the greatest protection. This would take the form of the development of adequate evacuation procedures and other procedures for improving resilience. These countries mostly have small populations and as such are not well represented at an international level, nor do they have the ability to provide assistance in the development of an international mitigation mission. Nevertheless, it would be important to include their voices if the OECD or the G8 were used as a forum for mitigation and deflection discussions. The five countries that face the greatest combined socio-economic risk (third column of Table 5) are likely to be most involved in any action to tackle the threat. They account for 41.4 per cent of all global casualties and 27.3 per cent of the global infrastructure loss but represent only 6.1 per cent of the global surface area at risk from a NEO impact. This disproportionate distribution of global loss is further evidence that ocean impact tsunamis represent the most significant threat.

\section{Implications for global vulnerability and the decision-making process}

The third column of Table 5 highlights the five countries that face the greatest combined socio-economic risk. There are implications for such a small number of countries being exposed to the major proportion of the overall risk. These five countries collectively have the most to lose in the event of an impact. Their proximity to all the major oceans (except the Southern Ocean), means that any ocean impact will strongly affect one or more of these countries. It could be argued that this would be sufficient motivation for these countries to be most involved in any action to tackle the threat. Currently the United States is leading efforts to catalogue threatening objects through the Spaceguard Survey, as well as having a high level of NEO mitigation research activity. Japan and Brazil both have long established space agencies and therefore potentially have the resources to plan and execute a mitigation mission. The Japan Aerospace Exploration Agency (JAXA) has demonstrated its capabilities though the Hayabusa mission (JAXA, 2006). China has a rapidly developing space activity, reviewed by Harvey (2004), having gone from first flight to first manned flight in a period of just four years. This implies that it could develop and launch a mitigation mission in a short time scale. India's space activity is also growing 
steadily with the recent launch of its first unmanned moon mission (ISRO, 2008).

\section{Conclusions}

Current NEO impact research is typically focused on individual topics (atmospheric flight, land impact or ocean impact) and, as such, there is generally no integration of these specialist topics to give a comprehensive view of the consequences of an impact. Some systems, such as the web-based simulator described in Collins et al (2005), provide public access to this research, but is limited, in this case, to determining an individual's personal risk from an event. NEOimpactor attempts to bridge the gap between the three specialist fields, combining many research areas into an accessible tool capable of giving an assessment of global vulnerability. Data output formats of the system have been designed with a non-specialist audience in mind to aid the dissemination of information. The primary data outputs are in the form of maps of global casualty and damage estimates. Further analysis is enabled by the simple manipulation of data sets using a spreadsheet package, such as Microsoft's Excel. Simulations performed by NEOimpactor have provided insight into a number of aspects of the NEO hazard. A summary of these is given below.

In this study, three event scale classifications are defined - Local, Regional and Global. These classifications are based on a combination of casualty and damage estimates, together with the geographical spread of affected populations. At an international level defining the scale of an event is important in determining the response required. Local events will primarily influence only one country, with casualties in the tens of thousands and damage of the order of one NIU. Regional events principally affect more than one country with casualties in the hundreds of thousands and damage in the tens of NIU. Global scale events are predominantly ocean impacts of large objects, with the possibility of affecting over a hundred nations, generating millions of casualties and thousands of NIU (hundreds of billions of dollars).

Land impacts are shown to exhibit an extreme range of consequences. This is due to the non-uniform distribution of human population, related to the distribution of natural resources. A direct hit on a conurbation may destroy everything, while the same impact $100 \mathrm{~km}$ away will have much less consequence. Ocean impacts, however, produce tsunamis which are very effective at propagating the damaging energy of impact over large distances. On average, for large bodies, the consequences resulting from an ocean impact will outweigh those from a land event, but what does 'large' mean in this context? All the simulation results demonstrate that the greatest risk from small objects arise when impacting land. They have minimal damaging effect when falling into the ocean. On the other hand, the principal threat from the fall of a large asteroid comes when it impacts the ocean. Consequently, there exists a 
transition radius at which the severity of the impact consequences switches from land to ocean impacts. Taking the assumptions used here of a spherical stony-iron asteroid, with an impact speed of $12000 \mathrm{~m} / \mathrm{s}$, we find the transition radius to be of the order of $30 \mathrm{~m}$, although the breadth of this study has been insufficient to be definitive about this conclusion.

A simple fragmentation study was performed using the NEOimpactor system, which indicated that multiple fragment impacts present a combined hazard typically greater than that of an equivalent energy, single impact event. This conclusion is of significance when considering asteroid mitigation by nuclear detonation, such as proposed in Gertsch et al (2007) and Patenaud (2007). While the aim of this methodology should be to reduce the asteroid to dust-grain sized particles, failure to achieve this will result in the fragmentation of the body into multiple medium size fragments, leading to potentially an increased threat to Earth. If multiple fragments were to impact the ocean, the effects of many tsunami are not cumulative and the consequence is effectively equivalent to just one small impact. However, multiple impacts on land increases the probability that one (or more) will hit a populated region directly, raising the casualty and damage potential significantly.

There are many factors which determine a particular country's vulnerability. The five principal ones found here are - total land area, size of human population, total infrastructure value, proximity to large oceans and length of coastline. The factors involved can be expanded to include the population density of coastal regions, the number of large sprawling urban conurbations, the 'land area to coastline length' ratio and the country's geographical morphology (whether it is land-locked or an island). Five countries have been highlighted as having the greatest combined human and infrastructure exposure to the risk. They are - China, United States, India, Japan and Brazil. However, the most vulnerable countries are those small island nations which face potential devastation in the event of a nearby impact. Land-locked countries present the lowest exposure to the hazard, as well as affording likely evacuation pathways, and can therefore be considered to be 'safest'.

A number of conclusions are drawn concerning the decision- and policymaking activities likely to be required in the event of a real impact threat. Firstly, regarding the nature of land and ocean impacts, there is confirmation of the general consensus that ocean impacts are the most significant impact threat. Secondly the capability is provided, through NEOimpactor, to model an asteroid's impact consequences along its predicted line of risk. This helps inform the mitigation strategists in attempting to plan the lowest risk mitigation mission. Thirdly, that the international community is informed regarding the nature of each country's vulnerability. The research presented here provides an indication of those counties that face the greatest risk. It could be argued that these nations, therefore, be called upon to lead the activity of 
creating the necessary technology and policy to combat the threat represented by Earth impacts of NEOs.

\section{Acknowledgements}

The authors gratefully acknowledge the sponsorship of the UK Science and Technology Facilities Council (STFC) and the School of Engineering Sciences, University of Southampton, UK. Without this support, the research presented in this article would not have been possible.

\section{References}

Boslough, M.B.E. and Crawford, D.A. (2008) Low-altitude airbursts and the impact threat. International Journal of Impact Engineering 35(12): 1441-1448.

Chyba, C.F. (1993) Explosions of small Spacewatch objects in the Earth's atmosphere. Nature 363(6431): 701-703.

Collins, G.S., Melosh, H.J. and Marcus, R.A. (2005) Earth impact effects program: A web-based computer program for calculating the regional environmental consequen-ces of a meteoroid impact on Earth. Meteoritics and Planetary Science 40(6): 817-840.

Galvez, A., Coradini, M. and Ongaro, F. (2003) The role of space missions in the assessment of the NEO impact hazard. Proceedings of the 54th International Astronautical Congress, Session C.2.3; 29 September-3 October, Bremen, Germany.

Gertsch, L., Baird, J. and Worsey, P. (2007) Blast designs for NEO destruction. Proceedings of the Planetary Defence Conference; 5-8 March 2007, Washington DC, http:// www.aero.org/conferences/planetarydefense/2007papers/P2-2--Gertsch-Paper.pdf, accessed 30 October 2008.

Harvey, B. (2004) China's Space Program: From Conception to Manned Spaceight. New York: Springer.

ISRO. (2008) Welcome to Indian space research organisation. Department of Space, Indian Space Research Organisation, http://www.isro.org/, accessed 1 December 2008.

JAXA. (2006) Hayabusa mission. Japanese Aerospace Exploration Agency, Institute of Space and Astronautical Science, http://www.isas.ac.jp/e/enterp/missions/hayabusa/ index.shtml, accessed 19 July 2006.

Maurrasse, F.J.M.R. and Sen, G. (1991) Impacts, tsunamis, and the Haitian cretaceoustertiary boundary layer. Science 252(5013): 1690.

NASA. (2008) Visible Earth: A catalog of NASA images and animations of our home planet. National Aeronautics and Space Administration, http://visibleearth.nasa.gov/, accessed 30 November 2008.

OECD. (2009) Organisation for economic co-operation and development, http://www .oecd.org/home/, accessed 17 January 2009.

Patenaud, S. (2007) Nuclear deection. Proceedings of the Planetary Defence Conference; 5-8 March 2007, Washington DC, http://www.aero.org/conferences/planetarydefense/ 2007papers/S2-2--Patenaud-Brief.pdf, accessed 30 October 2008.

Paxson, C. and Rouse, C.E. (2008) Returning to new Orleans after hurricane Katrina. American Economic Review 98: 38-42.

Pravec, P. and Harris, A.W. (2000) Fast and slow rotation of asteroids. Icarus 148(1): $12-20$. 
Rantucci, G. (1995) Geological Disasters in the Philippines: The July 1990 Earthquake and the June 1991 Eruption of Mount Pinatubo. Rome, Italy: Italian Ministry of Foreign Affairs.

Robinson, L. and Jarvie, J.K. (2008) Post-disaster community tourism recovery: The tsunami and Arugam Bay, Sri Lanka. Disasters 32(4): 631-645.

Savage, S.J. and Waldman, D. (2005) Broadband internet access, awareness, and use: Analysis of United States household data. Telecommunications Policy 29: 615-633.

Seed, H.B., Romo, M.P., Sun, J.I., Jaime, A. and Lysmer, J. (1988) The Mexico earthquake of September 19, 1985: Relationships between soil conditions and earthquake ground motions. Earthquake Spectra 4(4): 687-729.

Smit, J. (1999) Global stratigraphy of the cretaceous-tertiary boundary impact ejecta. Annual Review of Earth and Planetary Sciences 27: 75-113.

Stokes, G.H. and Yeomans, D.K. (2003) A study to determine the feasibility of extending the search for NEOs to smaller limiting diameters. Technical Report of a NASA science definition team, http://neo.jpl.nasa.gov/neo/neoreport030825.pdf, accessed November 2008.

Titov, V., Rabinovich, A.B., Mofjeld, H.O., Thomson, R.E. and Gonzalez, F.I. (2005) Ocean science: The global reach of the 26 December 2004 Sumatra tsunami. Science 309(5743): 2045-2048.

Ward, S.N. (2000) Tsunamis. In: R.A. Meyers (ed.) Encyclopaedia of Physical Science and Technology, Vol. 17. New York: Academic Press, p. 16.

Ward, S.N. and Asphaug, E. (2002) Impact tsunami - Eltanin. Deep-Sea Research Part II: Topical Studies in Oceanography 49(6): 1073-1079.

Ward, S.N. and Asphaug, E. (2003) Asteroid impact tsunami of 2880 March 16th. Geophysical Journal International 153(3): 6-10. 